\title{
A Smart Discrete Fourier Transform Algorithm Based Digital Multifunction Relay for Power System Protection
}

\author{
${ }^{*}$ P. Suresh Babu and ${ }^{* *}$ Dr. S. V. Jayaram Kumar
}

\begin{abstract}
Many large scale system blackouts involve relay misoperations. Technical advancements in relaying technology has replaced the concept of specific relay for specific action with digital multifunction relays where only single relay can perform different types of relaying functions with the help of digital processor programming. Traditional relay algorithms and settings need to be evaluated under variety of fault and nofault system wide scenarios to better understand the cause of misoperation of relays. The fault diagnosis algorithm also need to be developed to assure improved relaying performance and then evaluated under various system conditions. This paper presents a novel digital multifunction relay based on Smart Discrete Fourier Transform Algorithm (SDFTA) that derived from Discrete Fourier Transform (DFT) which can estimate exact magnitude of DC offset component and completely eliminates it from operating quantities during faults and also makes use of smoothing window to filter out noise if any. The proposed digital multifunction relay's performance is tested on 6-bus power system with the data generated by EMTP/PSCAD. The results had shown the efficient operation of the proposed SDFTA based digital multifunction relay.
\end{abstract}

Index Terms-Misoperation, digital protection, multifunction relays, filtering algorithm, decaying DC offset component, faults, phasor measurement, fault location.

\section{INTRODUCTION}

In power systems, high voltage transmission and distribution lines are vital links from generating plants to the end users along the most desirable path. Power systems are more vulnerable to contingencies and probability of power failure due to faults is higher. From the view of security, economics and quality of power feeding, it is necessary for protecting lines using relays. According to historical record, many large scale system-wide blackouts involve relay misoperations. So for each type of protection purpose it is necessary to install appropriate relay. Since it is not possible to optimize a standard relaying system to every protection need, digital multifunction protection schemes are proposed as they have features like reduction in cost, functional flexibility, adaptive relaying and self-checking capability. With the advantage of generally available hardware and software, having the speed and capability of digital relays necessary for protection application, it has now become

*Professor in EEE Dept., St. Ann's College of Engg. \& Tech, CHIRALA-523187, INDIA E-mail: sureshperli@yahoo.com

**Professor in EEE Dept., JNTU College of Engineering, Kukatpally, Hyderabad-500082, INDIA. E-mail: svjkumar101@rediffmail.com possible for the relay engineers to develop and implement custom protection solutions for those applications where standard packages may not provide the necessary flexibility or performance. When a fault is detected the protective relay must respond quickly to isolate the faulted line to preserve the stability of the rest of the system. However various conditions such as remote in feed currents, fault path resistance and shunt capacitance etc., degrade the performance.

Evaluation and improvement of existing relaying algorithms and settings as well as investigation of new techniques for relaying are very important for understanding and mitigating relay operations. The most common approach used by many researchers for studying relay algorithm performance is using a simple two machine system and limited fault scenarios. The proposed method cannot reflect the relay algorithm performance in the context of system wide disturbances but it is a software simulation for comprehensive study and analysis of different relaying functions under a variety of system wide disturbances.

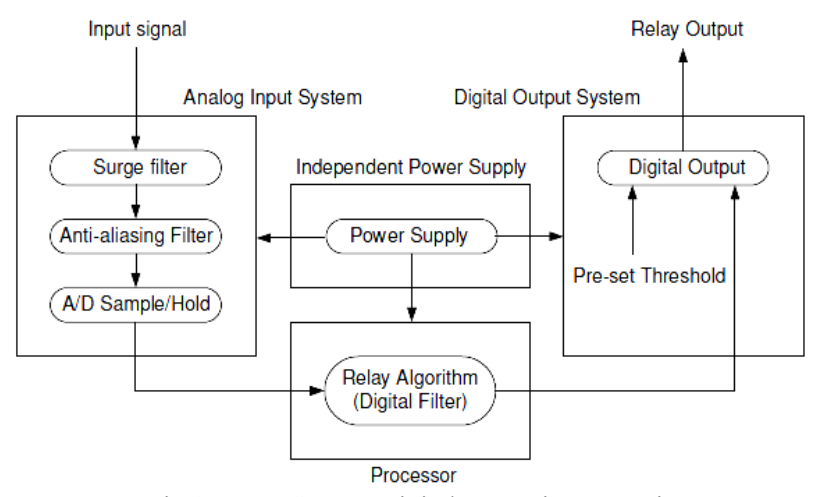

Fig.1 Power System Digital Protection scenario

When fault occurs, relay prevent involving area from growing. Hence correct action of relay is important in power system. However it is a fact that a line relay tends to over reach in presence of $\mathrm{DC}$ offset component in fault waveform. Therefor DC offset component has to be removed from these relay input waveforms. Digital multifunction relays are now being used in order to reduce installation, operation and maintenance costs. Input signals of protective relays contain distortion, which must be rejected to retain signal quantities of relevant interest. In addition, filters of digital relay must decompose the fundamental frequency component quickly and accurately for enhanced protection. Decaying dc seriously influence the accuracy and convergent speed of filter algorithms. 
Moreover, the time constant and amplitude of decaying dc of fault lines are unknown and associated with the fault resistance, fault position and fault beginning time. Discrete Fourier Transform is an excellent filtering algorithm capable of removing integer harmonics using simple computation. However, the voltage and current signals include serious harmonics and decaying dc during the fault period. The decaying $\mathrm{dc}$ and higher order harmonics severely inhibit the search for an accurate fundamental frequency signal and delay the convergence time. The fundamental frequency phasor estimation of the conventional DFT algorithm is not convergent within this time limit.

The present work focuses on presenting a novel digital multifunction relay based on novel Smart Discrete Fourier Transform Algorithm (SDFTA). It is derived from conventional Discrete Fourier Transform (DFT). This proposed algorithm keeps all the advantages of DFT and smartly avoids the defects resulting from frequency scales that cannot match signal characteristics. Noise is unavoidable in power system. Hence every algorithm applied to power system protection has to take noise into consideration while calculating fundamental frequency components that are given as relay inputs. At present more prevalent filtering methods are using smoothing windows. In general, use of smoothing windows is known to provide flexible solutions to filtering noise, but loses the ability of computing phasor. The proposed method also uses smoothing windows to filter out noise that can allay the phase shift and amplitude decay that caused by smoothing windows. The proposed method, Smart Discrete Fourier Transform Algorithm can estimate DC offset component exactly. In fact this algorithm obtains exact DC offset from faulted waveforms such that it can be eliminated. This is useful for fault detection and location. With this algorithm based proposed digital multifunction relay can work properly. Without influence of DC offset, relays obtain accurate frequency and phasor components from SDFT. Time constant of DC offset can be use in line parameter estimation after fault occurs. Uncertainty about the line parameter, which usually results in a significant error in the calculated fault parameters, can also be resolved by proposed algorithm. The exact fault angle of DC offset can also be calculated. All the data is very useful in extraction of exact fundamental frequency components of faulted signals for relaying applications.

\section{Proposed SdFt Filtering Algorithm}

The voltage and current signals may contain serious harmonics and decaying dc components during fault interval. The decaying DC seriously decreases the precision and convergence speed of fundamental frequency signal from DFT. In order to overcome the above problems, the proposed digital multifunction relay with SDFT algorithm can estimate the DC offset frequency and phasor from a faulted input operating signals. Since there are several components in a fault current signal, the algorithm first takes DC offset into consideration and uses smoothing windows to eliminate other components in a fault signal.

Consider any fault signal $f(t)$ with fundamental frequency components and decaying DC offset components can be expressed as

$$
\begin{aligned}
& f(t)=F \sin \left(\omega t+\phi_{1}\right)+F \sin \left(\phi_{2}\right) e^{-\alpha t} \\
& --1
\end{aligned}
$$

Where

$\mathrm{F}$ is the amplitude of the faulted signal

$\phi_{1}$ is the phase angle of the faulted signal

$\phi_{2}$ is the fault angle of the signal

$\alpha^{-1}=\tau$ is the time constant of the signal

Suppose $f(t)$ is sampled with a rate of sampling $(50 * \mathrm{~N})$

$\mathrm{Hz}$ to produce the sample set $\{f(k)\}$

$$
f(k)=F \sin \left(\omega \frac{k}{50 N}+\phi_{1}\right)+F \sin \left(\phi_{2}\right) \exp \left(-\alpha \frac{k}{50 N}\right)
$$

The signal $f(t)$ is conventionally represented by phasor complex number $\bar{f}$

$$
\bar{f}=F e^{j \phi_{1}}=F \cos \phi_{1}+j F \sin \phi_{1}
$$$$
----3
$$

Then $f(t)$ can be expressed as

$$
f(t)=\frac{\bar{f} e^{j \phi_{1}}-\bar{f}^{*} e^{-j \phi_{1}}}{2}+F \sin \left(\phi_{2}\right) \exp \left(-\alpha \frac{k}{50 N}\right)
$$
$----4$

Fundamental frequency components of Discrete Fourier transform of $\{f(k)\}$ is calculated from the following equation $\hat{f}_{r}=\frac{2}{N} \sum_{k=0}^{N-1} f(k+r) e^{-j 2 \pi k / N}$ $---5$

Taking frequency deviation $\omega=2 \pi(50+\Delta f)$ into consideration

$$
\begin{gathered}
\hat{f_{r}}=\frac{\bar{f}}{N} \sum_{k=0}^{N-1} e^{j 2 \pi(50+\Delta f) \frac{(k+r)}{50 N}} e^{-j 2 \pi \frac{k}{N}} \\
-\frac{\bar{f}^{*}}{N} \sum_{k=0}^{N-1} e^{j 2 \pi(50+\Delta f) \frac{(k+r)}{50 N}} e^{-j 2 \pi \frac{k}{N}} \\
+\frac{2 F \sin \left(\phi_{2}\right)}{N} \sum_{k=0}^{N-1} e^{-\alpha \frac{(k+r)}{50 N}} e^{-j 2 \pi \frac{k}{N}} \\
---6
\end{gathered}
$$

We can rearrange the Equn (6) as

$$
\begin{gathered}
\hat{f}_{r}=\frac{\bar{f}}{N} e^{j 2 \pi\left(1+\frac{\Delta f}{50}\right) \frac{r}{N}} \sum_{k=0}^{N-1} e^{j 2 \pi\left(\frac{\Delta f}{50}\right) \frac{r}{N}} \\
-\frac{\bar{f}^{*}}{N} e^{-j 2 \pi\left(1+\frac{\Delta f}{50}\right) \frac{r}{N}} \sum_{k=0}^{N-1} e^{-j 2 \pi\left(\frac{2+\Delta f}{50}\right) \frac{r}{N}} \\
+\frac{2 F \sin \left(\phi_{2}\right)}{N} e^{-\alpha \frac{r}{50 N} \sum_{k=0}^{N-1} e^{-\alpha \frac{k}{50 N}} e^{-j 2 \pi \frac{k}{N}}}
\end{gathered}
$$

Above Equn. (7) can be solved by the following identity

$$
\sum_{i=0}^{N-1}\left(e^{j \theta}\right)^{i}=\frac{\sin \frac{N \theta}{2}}{\sin \frac{\theta}{2}} e^{j(N-1) \frac{\theta}{2}}
$$


We can rearrange the Equn. (7) as

$$
\begin{array}{r}
\hat{f}_{r}=\frac{\bar{f}}{N} e^{j 2 \pi\left(1+\frac{\Delta f}{50}\right) \frac{r}{N}} \frac{\sin N \theta_{1}}{\sin \theta_{1}} e^{j(N-1) \theta_{1}} \\
-\frac{\bar{f}^{*}}{N} e^{-j 2 \pi\left(1+\frac{\Delta f}{50}\right) \frac{r}{N}} \frac{\sin N \theta_{2}}{\sin \theta_{2}} e^{j(N-1) \theta_{2}} \\
+\frac{2 F \sin \left(\phi_{2}\right)}{N} \frac{e^{-\frac{\alpha}{50}}-1}{e^{-\frac{\alpha}{50 N}-j-\frac{2 \pi}{N}}-1} e^{-\frac{\alpha r}{50 N}}
\end{array}
$$

$-9$

Where

$\theta_{1}=\frac{\pi \Delta f}{50 N}$ and $\theta_{2}=-\frac{\pi\left(2+\frac{\Delta f}{60}\right)}{N}$

By rearranging Equn. (9) we can get

$$
\begin{gathered}
\hat{f}_{r}^{(50+\Delta f)=\frac{\bar{f}}{N}} \frac{\sin N \theta_{1}}{\sin \theta_{1}} e^{j \frac{\pi}{50 N}(\Delta f(2 r+N-1)+100 r)} \\
-\frac{\bar{f}^{*}}{N} \frac{\sin N \theta_{2}}{\sin \theta_{2}} e^{j \frac{\pi}{50 N}(\Delta f(2 r+N-1)+100(r+N-1))} \\
+\frac{2 F \sin \left(\phi_{2}\right)}{N} \frac{e^{-\frac{\alpha}{50}}-1}{e^{-\frac{\alpha}{50 N}-j-\frac{2 \pi}{N}}-1} e^{-\frac{\alpha r}{50 N}}
\end{gathered}
$$$$
--10
$$

Let assign

$\frac{\bar{f}}{N} \frac{\sin N \theta_{1}}{\sin \theta_{1}} e^{j \frac{\pi}{50 N}(\Delta f(2 r+N-1)+100 r)}=A_{r}$

$--11$

$-\frac{\bar{f}^{*}}{N} \frac{\sin N \theta_{2}}{\sin \theta_{2}} e^{j \frac{\pi}{50 N}(\Delta f(2 r+N-1)+100(r+N-1))}=B_{r}$

$--12$

$\frac{2 F \sin \left(\phi_{2}\right)}{N} \frac{e^{-\frac{\alpha}{50}}-1}{e^{-\frac{\alpha}{50 N}-j-\frac{2 \pi}{N}}-1} e^{-\frac{\alpha r}{50 N}}=C_{\mathrm{r}}$

$--13$

Equn. (10) can be re written as

$\hat{f}_{r}=A_{r}+B_{r}+C_{r}$

So far the development of the algorithm of SDFT is the same as the traditional DFT method. So the SDFT can keep all advantages of DFT such as recursive and half-cycle computing manner. But in the DFT, it doesn't take DC offset into consideration and it assumes that the frequency deviation is small enough to be ignored. It always considers $\hat{f}_{r}=A_{r}$, so traditional DFT based methods incur error in estimating frequency and phasor when frequency deviates from nominal frequency $(50 \mathrm{~Hz})$ or DC offset is present. If we want to obtain exact solution, we must take $B$, and $C$, into consideration. Then we define

$a=e^{j\left(\frac{\pi}{50 N}(2 \Delta f+100)\right)}$

$--15$

$b=e^{-\alpha / 50 N}$

$-16$

$-$
From Equn (10) following relations are obtained

$A_{r+1}=A_{r} . a$

$--17$

$B_{r+1}=B_{r} \cdot a^{-1}$

$--18$

$C_{r+1}=C_{r} \cdot b$

$--19$

Then

$\hat{f}_{r+1}=A_{r+1}+B_{r+1}+C_{r+1}=A_{r} \cdot a+B_{r} \cdot a^{-1}+C_{r} \cdot b$

$-20$

$\hat{f}_{r+2}=A_{r+2}+B_{r+2}+C_{r+2}=A_{r+1} \cdot a+B_{r+1} \cdot a^{-1}+C_{r+1} \cdot b$

$-21$

Equn.(14) is multiplied both sides with ' $b$ ' and subtract from Equn (20) gives

$\hat{y}_{r}=\hat{f}_{r+1}-\hat{f}_{r} b=A_{r}(a-b)+B_{r}\left(a^{-1}-b\right)$

$--22$

$\hat{y}_{r+1}=\hat{f}_{r+2}-\hat{f}_{r+1} b=A_{r+1}(a-b)+B_{r+1}\left(a^{-1}-b\right)$

$-23$

$\hat{y}_{r+2}=\hat{f}_{r+3}-\hat{f}_{r+2} b=A_{r+2}(a-b)+B_{r+2}\left(a^{-1}-b\right)$

$-24$

We can rearrange Equns.(22), (23) and (24) as

$\hat{y}_{r+1} a-\hat{y}_{r}=A_{r}\left(a^{2}-1\right)(a-b)$

$-25$

$\hat{y}_{r+2} a-\hat{y}_{r+1}=A_{r+1}\left(a^{2}-1\right)(a-b)$

$-26$

Equn (25)/ equn (26) gives

$a=\frac{A_{r+1}}{A_{r}}=\frac{\hat{y}_{r+2} a-\hat{y}_{r+1}}{\hat{y}_{r+1} a-\hat{y}_{r}}=\frac{\hat{y}_{r+3} a-\hat{y}_{r+2}}{\hat{y}_{r+2} a-\hat{y}_{r+1}}$

27

Put Equn.(23) \& Equn.(24) in Equn.(27)

$$
\begin{gathered}
{\left[\hat{f}_{r+2}\left(\hat{f}_{r}+\hat{f}_{r+2}\right)-\hat{f}_{r+1}\left(\hat{f}_{r+1}+\hat{f}_{r+3}\right)\right] b^{2}+} \\
{\left[\hat{f}_{r+3}\left(\hat{f}_{r}+\hat{f}_{r+2}\right)-\hat{f}_{r+1}\left(\hat{f}_{r+2}+\hat{f}_{r+4}\right)\right] b+} \\
{\left[\hat{f}_{r+3}\left(\hat{f}_{r+1}+\hat{f}_{r+3}\right)-\hat{f}_{r+2}\left(\hat{f}_{r+2}+\hat{f}_{r+4}\right)\right]=0}
\end{gathered}
$$

$-28$

Solve Equn.(28) to obtain 'b'. From the definition of ' $b$ ' in Equn (16) we can obtain the exact solution of the time constant.

$$
\tau=\frac{1}{50 N \log b}
$$

$-29$

Equn.(27) can be rearranged as

$\hat{y}_{r+1} a^{2}-\left(\hat{y}_{r}+\hat{y}_{r+2}\right) a+\hat{y}_{r+1}=0$

--30

Solve Equn.(30) to obtain 'a'. From the definition of 'a' in Equn. (15) we can get the exact solution of the frequency.

$f=50+\Delta f=\cos ^{-1}\{\operatorname{Re}(a)\} \frac{50 N}{2 \pi}$

$-31$

From Equn.(29) and Equn.(3I), it is observed that SDFT can provide exact time constant and frequency 
using $\hat{f}_{r}, \hat{f}_{r+1}, \hat{f}_{r+2}, \hat{f}_{r+3}$ and $\hat{f}_{r+4}$ in the absence of noise. Moreover, we can estimate phasor and fault angle after getting exact time constant and frequency by the following equations:

$$
A_{r}=\frac{\hat{y}_{r+1} a-\hat{y}_{r}}{\left(a^{2}-1\right)(a-b)}
$$

32

$$
F=a b s\left(A_{r} \frac{N \sin \theta_{1}}{\sin \left(N \theta_{1}\right)}\right)
$$

33

$$
\phi_{1}=\operatorname{angle}\left(A_{r} e^{-j \theta_{1}(N-1)}\right)
$$

34

$$
C_{r}=\frac{a^{2} \hat{f}_{r+1}-a\left(\hat{f}_{r}+\hat{f}_{r+2}\right)+\hat{f}_{r+1}}{(a-b)(a b-1)}
$$

35

$$
\phi_{2}=\sin ^{-1}\left(\frac{C_{r} N}{2 X} \frac{e^{-\frac{\alpha}{50 N}-j \frac{2 \pi}{N}}-1}{e^{-\frac{\alpha}{50}}-1}\right)
$$

$--36$

Furthermore, we take noise into consideration and use smoothing windows to filter noise. Consider a sampled set $\{f(k)\}$ to be a filtered set $\{z(k)\}$ by a smoothing window

$$
\begin{aligned}
& \left\{\left.S W_{(m)}\right|_{s_{1}, s_{2}, s_{3} \ldots \ldots s_{m}}\right. \text { with window size 'm'. } \\
& z(k)=\sum_{i=1}^{m} s_{i} f(k+i-1)
\end{aligned}
$$

$---37$

Moreover, the DFT of $\{z(k)\}$ is given by

$$
\begin{aligned}
\hat{z}_{r} & =\frac{2}{N} \sum_{k=0}^{N-1} z(k+r) e^{-j \frac{2 \pi k}{N}} \\
& =\frac{2}{N} \sum_{k=0}^{N-1}\left[\sum_{i=1}^{m} s_{i} f(k+r+i-1)\right] e^{-j \frac{2 \pi k}{N}} \\
& =\sum_{i=1}^{m} s_{i}\left[\frac{2}{N} \sum_{k=0}^{N-1} f(k+r+i-1) e^{-j \frac{2 \pi k}{N}}\right] \\
& =\sum_{i=1}^{m} s_{i} \hat{f}_{r+i-1}
\end{aligned}
$$$$
---38
$$

From the definition of Equn.(14), we can obtain:

$$
\begin{aligned}
& \hat{z}_{r}=A_{r}\left(s_{1}+s_{2} a+\ldots \ldots+s_{m} a^{m-1}\right) \\
& +B_{r}\left(s_{1}+s_{2} a^{-1}+\ldots \ldots+s_{m} a^{-(m-1)}\right) \\
& \quad+C_{r}\left(s_{1}+s_{2} b+\ldots \ldots+s_{m} b^{(m-1)}\right)
\end{aligned}
$$

The relations of Equn.(17), Equn.(18) and Equn.(19) are still kept in Equn.(39). Therefore, the same steps from Equn.(20) to Equn.(33) can be used in Equn.(39). Hence we can estimate time constant and frequency without modifying equations, but we have to do some change in Equn.(32) and Equn.(35) when we estimate phasor and fault angle.
$A_{r}=\frac{\hat{y}_{r+1} a-\hat{y}_{r}}{\left(a^{2}-1\right)(a-b)\left(s_{1}+s_{2} a+\ldots \ldots+s_{m} a^{m-1}\right)}$

$---40$

Where

$\hat{y}_{r}=\hat{z}_{r+1}-\hat{z}_{r} b$

$\hat{y}_{r+1}=\hat{z}_{r+2}-\hat{z}_{r+1} b$

$C_{r}=\frac{a^{2} \hat{f}_{r+1}-a\left(\hat{f}_{r}+\hat{f}_{r+2}\right)+\hat{f}_{r+1}}{(a b-1)(a-b)\left(s_{1}+s_{2} b+\ldots \ldots+s_{m} b^{m-1}\right)}$

$---41$

The phasor obtained from Equn.(40) and fault angle obtained from Equn.(41) will allay the phase shift and amplitude decay caused by smoothing windows.

\section{RELAY LOGICS}

The relay logic used in this work has two levels: Function level and Unit level. At the Function level, the logic checks for the occurrence of a fault using the predefined trip criteria that discriminate between fault and load condition. If fault is detected, the relay logic calculates the time that has to elapse before a trip command can be issued from the current instant. At unit level, the outputs of the function level logic are correlated to generate a trip command after the lapse of the lowest of the time to trip values of the individual functions if the corresponding trip criterion still remains satisfied.

The Function Level Logic implementation is as follows

\section{A. Distance Function Logic:}

Distance protection is applied on radial and transmission lines to identify and discriminate faults along the protected segment of the line, and provide system protection by isolating the faulted segment. The distance to the location of the fault along the line can be expressed as a percentage $m$ of the total length of the line. It has been shown that the length of the line between the location where measurements are taken and the location of the fault is measurable using voltages and currents obtained at the beginning of the line.

For a line-to-line fault between phases $\mathrm{A}$ and $\mathrm{B}$, the distance to the fault can be determined as:

$Z_{m}=m Z_{1}=\frac{V_{A}-V_{B}}{I_{A}-I_{B}}$

$--42$

Where

$\mathrm{Z}_{1}$ is the positive-sequence impedance of the line.

Using a similar approach, the impedance of the line to the location of the fault during a line-to-ground fault on phase A can be expressed as:

$Z_{m}=m Z_{1}=\frac{V_{A}}{I_{A}-k I_{o}}$

$-43$

$k=\frac{Z_{0}-Z_{1}}{Z_{0}}$

Where

$I_{o}$ is zero-sequence current and $Z_{0}$ is Zero sequence impedance.

The Function logic supports two commonly used operating Circular and Quadrilateral characteristics of 
conventional impedance relays. The Function Logic implements a three stepped distance protection by accepting three such characteristics, one for each zone. Conventionally, the instantaneous reach of the Distance function is set to $85 \%$ of the line length to allow a margin for the relay overreach due to the DC component of the fault current. The present scheme allows the extension of this setting to $95 \%$ of the line length. This is possible because the proposed method used effectively suppresses the DC offset component and harmonics in the fault signal and makes the distance function less prone to over-reaching error.

A distance relay that uses the above equations to determine the location of a fault is called a self-polarized impedance relay. The accuracy of the self-polarized distance relay is affected by infeeds and by the resistance of the fault. Therefore, its application is recommended to transmission lines with limited infeed or to radial lines. Depending on the measured impedance, different zones of protection can be set up by comparing the value of $Z_{m}$ to predefined numbers. As seen from the equations representing the measured apparent impedance to the fault, both currents and voltages are required. Using the open-system protection and control approach, both the overcurrent and distance elements can be done by the same clustered relaying installation. The circuit breaker operation is achieved by the same unified process communications. The radial line fault tests are conducted on 6- Bus test system shown in Fig.3

\section{B. Overcurrent Function Logic:}

The overcurrent protection is based on the amplitude of the current that flows at the location of the protective relay. It is a widely used protective function and due to its simplicity and reliability it has been maintained in new digital and microcomputer based solutions. The monitored value could be the RMS or peak value of the measured current. It is usually applied on a per phase basis, which means that at least 3 relays, preferably 4 with the one in the neutral, are needed to provide protection against all types of faults. It is a standard protective function on radial lines when the maximum load and minimum fault current are sufficiently distinct to provide enough margin for the relay to discriminate between load and fault currents.

The operation of the overcurrent protective function can be made instantaneously, as soon as the amplitude was determined to exceed a predefined value. In this case, the protective function is instantaneous overcurrent, and is represented by the IEEE function 50 .

The following condition thus results for the setting of the pick-up current

$$
I_{F \min }>I_{\text {Pick-up }}>I_{L \max }
$$$$
---44
$$

IF min -- minimum fault current at the relay location for a fault at limit of the protected zone

IL max -- maximum permissible load current of the protected line

Ipick-up -- relay (set) pick-up current

On radial lines, the fault current amplitude is inversely proportional to the distance between the location of the relay and that of the fault. Inverse-time overcurrent relays offer a fast response at high current values, and slower, delayed response to more distant fault events that result in smaller fault currents. The inverse-time characteristic of these relays permits the time coordination between protective devices along the radial lines. The inverse-time overcurrent characteristic is represented by the IEEE protective function 51 .

The over current tests are done using Inverse-time over current relay function given in the following Equations (45) and (46):

$$
t=\frac{C K}{\left(I^{n}-1\right)}
$$$$
--45
$$

I is input current

$\mathrm{K}$ is time multiplier setting

$\mathrm{C} \& \mathrm{n}$ are the constants determining inverseness.

Operating characteristics of Inverse-time over current relay is obtained with the following equation

$$
t=T D \frac{0.014}{M^{2}-1}
$$

TD is the time-dial setting,

$\mathrm{M}$ is the multiple of pickup current.

In present work this setting is based on the peak values of the incoming fault current. If the relay current drops below the set value before the lapse of the time to trip, the function logic resets itself automatically and jumps to check other relay function. With a pickup current $\mathrm{Ip}=2$ [p.u.] and TD = 0.5 . The exponential of $M$ is rounded and set equal to 2 in order to obtain a very fast response from the inverse-time overcurrent characteristic curves of the relay.

\section{HIGH ACURACY FAULT LOCATOR}

When the fault point is determined by measuring the impedance using local voltages and currents, the measurement error is increased as a result of the phase difference between the local and remote currents flowing into the fault point. The fault locator shown in Fgi.2, incorporated in present Digital multifunction relay, measures the distance to fault using local and remote voltages and currents. The fault point is calculated using the following Equation

$D=\frac{V_{A}-V_{B}+Z I_{B}}{Z\left(I_{A}+I_{B}\right)}$

$---47$

where,

D : Distance from Relay to the fault point

VA, IA : Local terminal voltage/current

$\mathrm{V}_{\mathrm{B}}$, Ів: Remote terminal voltage/current

$\mathrm{Z}$ : Line impedance

Relay at 


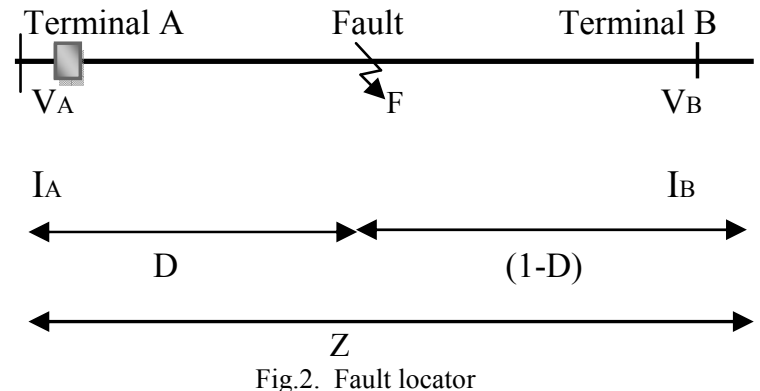

\section{MISOPERATION PREVENTIVE MEASURES OF PROPOSED RELAY}

Proposed Digital multifunction relay can increase the coverage of the instant operation zone and improve sensibility by taking proper precautions as below.

\section{a) Voltage start element}

In order to prevent relay operating incorrectly on open circuit, a voltage start element is used to increase reliability of the relay. The voltage start element includes a negative over voltage element and a phase to phase under voltage element.

\section{i) Negative over voltage element}

Negative over voltage element can pick up when unsymmetrical fault occurs. The criterion of negative over voltage element is

$V_{2}>V_{o p, 2}$

$---48$

where $V_{2}$ is the negative voltage.

$V_{o p, 2}$ is the operating value of the negative over voltage element:

$V_{o p, 2}=K_{r e l} V_{u m b-2}$

$---49$

where

$V_{u m b-2}$ is the maximal unbalanced negative voltage

$K_{r e l}$ is the reliable coefficient.

The relay will start when the Equn.(49) is met and drop off when it is not.

\section{ii) Phase-Phase under voltage element}

Phase-phase under voltage element is used for symmetrical fault. The criterion of the phase-phase under voltage element is

$V_{\phi \phi}<V_{o p, p}$

$---50$

Where $V_{\phi \phi}$ is the phase-phase voltage: $V_{A B}, V_{B C}, V_{C A}$

$V_{o p, p}$ is the operating value of phase-phase under voltage element:

$V_{o p, p}=\frac{(0.9 \rightarrow 0.95) V_{e}}{K_{r e l}}$
Where $V_{e}$ is the normal phase-phase voltage; $K_{r e l}$ is the reliable coefficient.

To prevent Phase-phase under voltage element from start under condition of power swing, a 1 cycle pre-fault (swing) voltage is used as the normal phase-phase voltage.

The relay will start when the condition in Equn.(51) is met, will drop off when it is not.

\section{b) Phase selector and block element}

The voltage start element can prevent operation incorrectly when open circuit occurs. However when fault occurs in successive operation zone, the voltage start element will pick up. Because the remote end breaker will trip first, healthy phase relay of the healthy line may operate incorrectly.

To prevent this, a phase selection element is used to block the potential operation of the health phase.

The criteria is

$\left|I_{1, i}\right|<K_{r e l} \cdot \max \left\{\left|I_{1, i}\right|\right\}$

Where $I_{1, i}$ is current of any line

$K_{r e l}$ is the reliable coefficient.

Any phase current meeting equation (52) is a healthy phase and should be blocked.

\section{IMPLEMENTATION \& TEST RESULTS}

The testing of the proposed SDFT algorithm based digital multifunction relay is done on a two functions applied to power system protective applications with increasing complexity. The composite system shown in Fig. 3 is used to test the new process-bus digital relaying solution. The system has a radial line, similar to structures used to supply large industrial loads on dedicated feeders. There are numerous radial sub-transmission systems installations to supply large industrial loads. Operation of these dedicated sub-transmission lines is done at medium voltage, usually between 66 and $132 \mathrm{kV}$. Due to the large load, the system needs to be relatively strong to avoid the voltage flickers during sudden load changes. The resulting separations between fault and load currents make these lines good candidates for over current protection. The radial line structure is also well suited for impedance protection. The operating voltage of the radial line in the test system is 132 $\mathrm{kV}$, and the fault location is marked with $\mathrm{F}$.

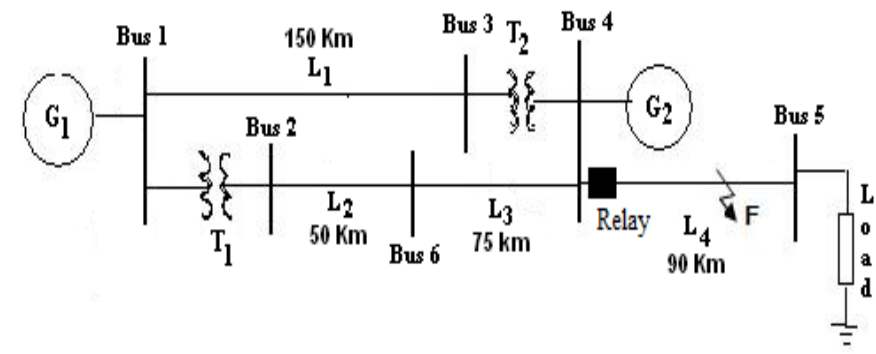

Fig.3. 6-Bus Test System 


\section{Test System Data}

\section{Lines Data:}

\begin{tabular}{|c|c|c|c|c|c|c|}
\hline Line & $\begin{array}{c}\text { Voltage } \\
(\mathbf{K V})\end{array}$ & $\begin{array}{c}\mathbf{R}_{\mathbf{1}} \\
(\mathbf{\Omega})\end{array}$ & $\begin{array}{c}\mathbf{R}_{\mathbf{0}} \\
(\mathbf{\Omega})\end{array}$ & $\mathbf{L}_{\mathbf{1}}(\mathbf{H})$ & $\mathbf{L}_{\mathbf{0}}(\mathbf{H})$ & $\begin{array}{c}\text { Length } \\
(\mathbf{K m})\end{array}$ \\
\hline $\mathbf{L}_{\mathbf{1}}$ & 220 & 0.602 & 11.5 & 0.03 & 0.139 & 150 \\
\hline $\mathbf{L}_{\mathbf{2}}$ & 132 & 0.318 & 9.66 & 0.0233 & 0.0825 & 50 \\
\hline $\mathbf{L}_{\mathbf{3}}$ & 132 & 0.255 & 7.33 & 0.0187 & 0.0825 & 75 \\
\hline $\mathbf{L}_{\mathbf{4}}$ & 132 & 0.446 & 1.35 & 0.0327 & 0.144 & 90 \\
\hline
\end{tabular}

\section{Generator Data:}

\begin{tabular}{|l|c|c|c|l|}
\hline & $\begin{array}{c}\text { Voltage } \\
(\mathbf{K V})\end{array}$ & $\begin{array}{c}\text { Rating } \\
\text { GVA }\end{array}$ & $\begin{array}{c}\mathbf{X} / \mathbf{R} \\
\text { ratio }\end{array}$ & $\begin{array}{c}\text { Winding } \\
\text { connection }\end{array}$ \\
\hline Generator- $\mathrm{G}_{1}$ & $220 \angle 0^{\circ}$ & 10 & 5 & Star-Grounded \\
\hline Generator- $\mathrm{G}_{2}$ & $132 \angle-10^{\circ}$ & 1.5 & 2 & Star- rounded \\
\hline
\end{tabular}

\section{Transformers $\mathbf{T} 1 \& \mathbf{T}_{2}$ Data:}

\section{Rating: 250MVA}

Impedance of windings $\mathrm{Z} 1=\mathrm{Z}_{2}=(2+\mathrm{j} 80) X 10^{-3} \mathrm{p}$. u.

Magnetizing branch impedance $Z_{\mathrm{m}}=(500+\mathrm{j} 500) \mathrm{p}$. $\mathrm{u}$

Winding Connections of $\mathrm{T}_{1} \stackrel{\mathrm{Y}}{\underline{\mathrm{Y}} / \mathrm{Y}}$

Winding Connections of $\mathrm{T}_{2} \mathrm{Y} / \mathrm{Y}_{\underline{Z}}$

Load Data: 3-Phase, 132KV，20 $\angle 1.145^{\circ}$ MVA

\section{i) Case -1 : LG Fault}

Single line to ground fault was created on Phase-A of the transmission line $\mathrm{L}_{4}$ which is in between Bus 4 and Bus 5 . The fault was incepted at an angle of $0^{\circ}$. The distance of fault creation from relay location is $40 \mathrm{Km}$. Pre-set value of Pick up time of over current relay is less than $24 \mathrm{~ms}$. Pre-set value of Trip time of distance relay is less than $16 \mathrm{~ms}$. Simulation results are shown in fig.4 (a), 4(b) and Table-I. In case of Distance function, the fault was identified at $0.00372 \mathrm{sec}$ and time of trip signal generation is $0.0094 \mathrm{sec}$.

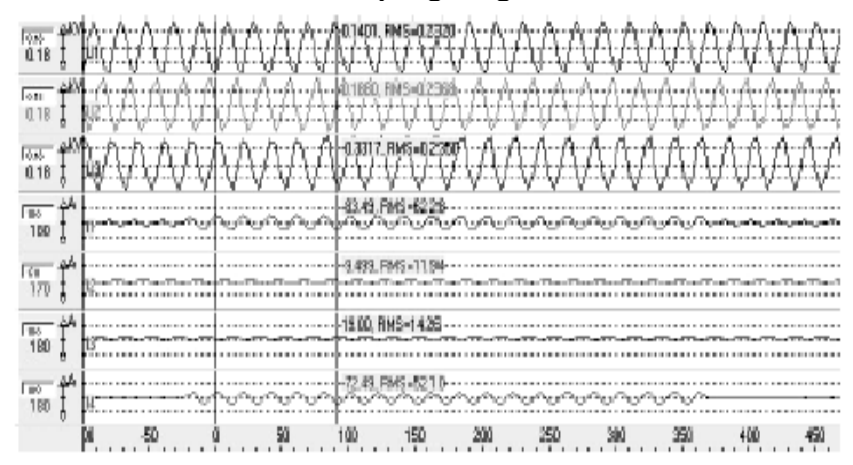

Fig. 4(a) : Voltage and Current waveforms for Phase-A to Ground Fault
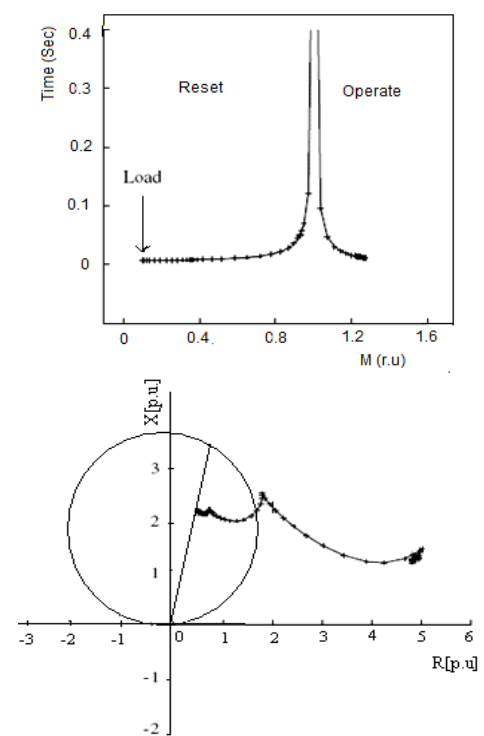

Fig4(b). Overcurrent protection performance and Impedance Evolution for L-G fault at location $\mathrm{F}$

\section{ii) Case -2 : L-L Fault}

Double line to ground fault was created on Phase-A and Phase- $\mathrm{B}$ of the transmission line $\mathrm{L}_{4}$ which is in between Bus 4 and Bus 5. The fault was incepted at an angle of $30^{\circ}$. The distance of fault creation from relay location is $65 \mathrm{Km}$. Preset value of Pick up time of over current relay is less than $24 \mathrm{~ms}$. and pre-set value of trip time of distance relay is less than $16 \mathrm{~ms}$.

Simulation results are shown in fig.5(a), 5(b) and Table-I. In case of Distance function, the fault was identified at $0.00495 \mathrm{sec}$ and time of trip signal generation is $0.0068 \mathrm{sec}$.

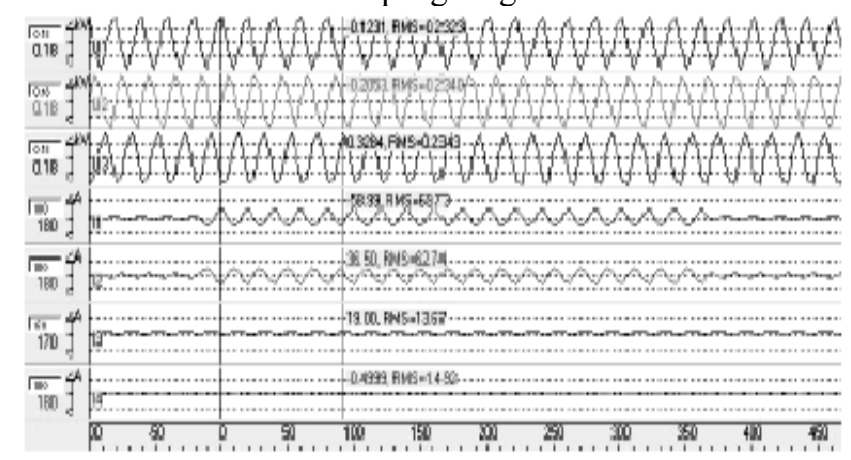

Fig.5(a): Voltage and Current waveforms for Phase-A to Phase- B Fault
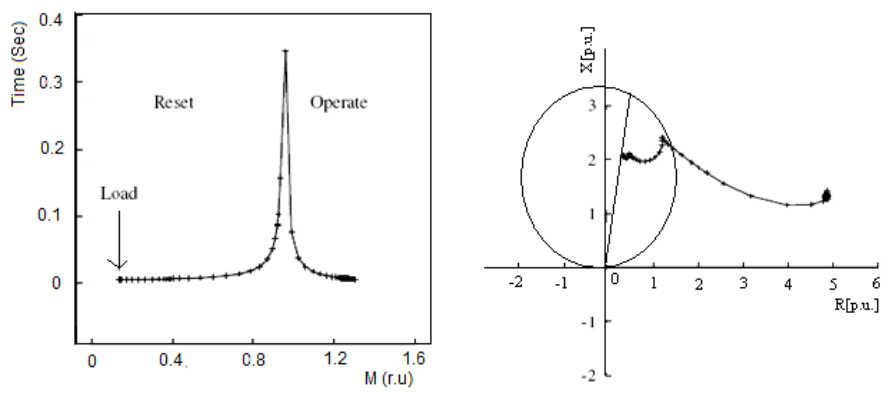

Fig5(b). Overcurrent protection performance and Impedance Evolution for $L-L$ fault at location $F$ 


\section{iii) Case -3 : L-L-L Fault}

Triple Line fault was created on the transmission line $\mathrm{L}_{4}$ which is in between Bus 4 and Bus 5. The fault was incepted at an angle of $45^{\circ}$. The distance of fault creation from relay location is $80 \mathrm{Km}$. Pre-set value of Pick up time of over current relay is less than $24 \mathrm{~ms}$ and pre-set value of trip time of distance relay is less than $16 \mathrm{~ms}$. Simulation results are shown in Fig.6 (a), 6(b) and Table-I. In case of Distance function, the fault was identified at $0.00167 \mathrm{sec}$ and time of trip signal generation is $0.0073 \mathrm{sec}$.

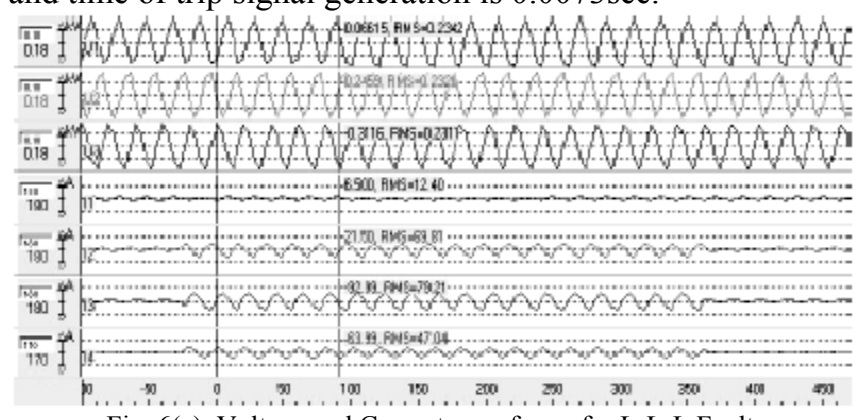

Fig. 6(a): Voltage and Current waveforms for L-L-L Fault
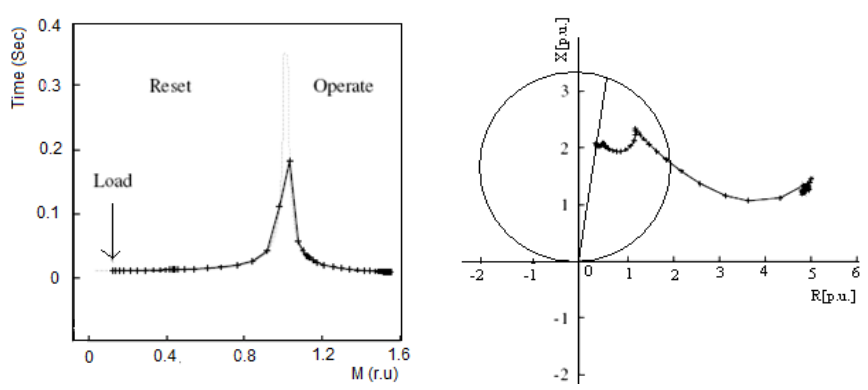

Fig6 (b). Overcurrent protection performance and Impedance Evolution for L-L-L fault at location $\mathrm{F}$

The Performance results of proposed SDFT based digital multifunction relay for all types of faults are tabulated in Table-I

\section{CONCLUSIONS}

In this paper, the calculation of the relay setting values has been included and some types of faults, based on severity and probability of occurrence, which may occur in the power system have been tested. The proper operation of the proposed multifunction relay has also been demonstrated. In presence of a fault within the zone of protection, the measured impedance is within the set boundaries of the characteristic. When the corresponding delay time is reached, trip signal is generated which is within the pre-set value. It has been shown the successful functionality of the proposed relay. This work can be extended to study the performance of this relay in presence of power system oscillations, for source impedance variations and for evolving faults, e.g., faults starting as phase to ground fault, but developing to double-phase to ground fault.

TABLE-I
\begin{tabular}{|l|c|c|c|}
\hline \multicolumn{1}{|c|}{ Parameter/ Item } & L-G & L-L & L-L-L \\
\hline Type of the fault created on Phase & A-G & A-B & A-B-C \\
\hline Fault created distance in Km & 40 & 65 & 80 \\
\hline $\begin{array}{l}\text { Time of Fault created instant in } \\
\text { Sec }\end{array}$ & 0.01602 & 0.01467 & 0.0134 \\
\hline Fault inception angle in Degrees & 0 & 30 & 45 \\
\hline
\end{tabular}

\begin{tabular}{|l|c|c|c|}
\hline Fault identification time in Sec & 0.0204 & 0.01717 & 0.0147 \\
\hline Pick up time of relay in Sec & 0.0229 & 0.02030 & 0.0172 \\
\hline Trip time in Sec & 0.02665 & 0.02283 & 0.0190 \\
\hline Type of Fault displayed as Phase & A-G & A-B & A-B-C \\
\hline $\begin{array}{l}\text { Distance of fault computed from } \\
\text { relay location to fault point in Km }\end{array}$ & 39.726 & 64.19 & 78.97 \\
\hline $\begin{array}{l}\text { \%Error in fault location } \\
\text { calculation }\end{array}$ & $0.625 \%$ & $1.246 \%$ & $\begin{array}{c}0.837 \\
\%\end{array}$ \\
\hline
\end{tabular}

\section{REFERENCES}

[1] J. F. M. Arguelles, M. A. Z. Arrieta, J. L. Dominguez, B. L. Jaurrieta,and M. S. Benito, "A new method for decaying dc offset removal for digital protective relays," Elect. Power Syst. Res., vol. 76, pp. 194-199, Jun. 2006.

[2] T. S. Sidhu, X. Zhang, and V. Balamourougan, "A new half-cycle phasor estimation algorithm," IEEE Trans. Power Del., vol. 20, no. 2,pt. 2, pp. 1299-1305, Apr. 2005.

[3] C.S.Chen, C. W. Liu, and J. A. Jiang, "Application of combined adaptive Fourier filtering technique and fault detector to fast distance protection,"IEEE Trans.Powe Del., vol.21, no.2, pp.619-626,Apr. 2006.

[4] J. C. Gu and S. L. Yu, "Removal of dc offset in current and voltage signals using a novel Fourier filter algorithm," IEEE Trans. Power Del.,vol. 15, no. 1, pp. 73-79, Jan. 2000.

[5] T.S.Sidhu, X.Zhang, F.Albasri, and M.S. Sachdev, "DiscreteFouriertransform-based technique for removal of decaying dc offset from phasor estimates," Proc. Inst Elect Eng.,Gen. Trans.Distrib.,vol.150, pp. 745-752, Nov. 2003.

[6] Nan Zhang, M Kezunovic," A study of synchronized sampling based failure location algorithm performance under power swing and stepout-condition"St.Petersburgpower-tech'05, St.Peterburg,Russia ,June'05

[7] D.Ristanovic, S.Vasilic" Design and implementation of Scenarios for evaluating and testing distance relays" North American power Symposium- NAPS, College station ,Texas, Oct-2001.

[8] IEEE/PSRC Working Group 13, "Transmission Protective Relay System Performance Measuring Methodology," September 1999.

[9] J. D. McDonald, "Substation Automation: IED Integration and Availability of Information" IEEE Power \& Energy, Vol. 1, No. 2, March/April 2003, pp. 22-31.

[10] Gabriel Benmouyal "Removal of Dc offset in current waveforms using digital mimic filtering" IEEE Power delivery, Vol.10, No.2, April 1995, pp. 621-630

[11] Adly A. Girgis, A. A.Sallam, A. Karim El-Din” An Adaptive Protection schemes for advanced series compensated (ASC) transmission line", IEEE Trans. On Power delivery, Vol.13,No.2, April 1998, PP.414-420.

[12] http://www.mantatest.com/Protection\%20Library.html

[13] http://www.abb.com/substationautomation 